\title{
BMJ Open Bi-modal stimulation in the treatment of tinnitus: a study protocol for an exploratory trial to optimise stimulation parameters and patient subtyping
}

\author{
Shona D'Arcy, ${ }^{1}$ Caroline Hamilton, ${ }^{1}$ Stephen Hughes, ${ }^{1}$ Deborah A Hall, ${ }^{2,3}$ \\ Sven Vanneste, ${ }^{4}$ Berthold Langguth, ${ }^{5,6}$ Brendan Conlon ${ }^{1,7,10,8}$
}

To cite: D'Arcy S, Hamilton C, Hughes $\mathrm{S}$, et al. Bi-modal stimulation in the treatment of tinnitus: a study protocol for an exploratory trial to optimise stimulation parameters and patient subtyping. BMJ Open 2017;7:e018465. doi:10.1136/ bmjopen-2017-018465

- Prepublication history for this paper is available online. To view these files, please visit the journal online (http://dx.doi. org/10.1136/bmjopen-2017018465).

Received 4 July 2017 Revised 10 August 2017 Accepted 23 August 2017

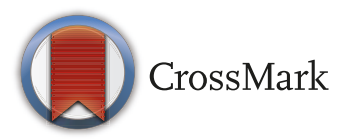

For numbered affiliations see end of article.

Correspondence to Dr Shona D'Arcy; shona.darcy@neuromoddevices. com

\section{ABSTRACT}

Introduction Tinnitus is the perception of sound in the absence of a corresponding external acoustic stimulus. Bimodal neuromodulation is emerging as a promising treatment for this condition. The main objectives of this study are to investigate the relevance of interstimulus timing and the choices of acoustic and tongue stimuli for a proprietary bimodal (auditory and somatosensory) neuromodulation device, as well as to explore whether specific subtypes of patients are differentially responsive to this novel intervention for reducing the symptoms of chronic tinnitus.

Methods and analysis This is a two-site, randomised, triple-blind, exploratory study of a proprietary neuromodulation device with a pre-post and 12-month follow-up design. Three different bimodal stimulation parameter sets will be examined. The study will enrol 342 patients, split 80:20 between two sites (Dublin, Ireland and Regensburg, Germany), to complete 12 weeks of treatment with the device. Patients will be allocated to one of three arms using a stepwise stratification according to four binary categories: tinnitus tonality, sound level tolerance (using loudness discomfort level of $<60 \mathrm{~dB}$ $\mathrm{SL}$ as an indicator for hyperacusis), hearing thresholds and presence of a noise-induced audiometric profile. The main indicators of relative clinical efficacy for the three different parameter sets are two patient-reported outcomes measures, the Tinnitus Handicap Inventory and the Tinnitus Functional Index, after 12 weeks of intervention. Clinical efficacy will be further explored in a series of patient subtypes, split by the stratification variables and by presence of a somatic tinnitus. Evidence for sustained effects on the psychological and functional impact of tinnitus will be followed up for 12 months. Safety data will be collected and reported. A number of feasibility measures to inform future trial design include: reasons for exclusion, completeness of data collection, attrition rates, patient's adherence to the device usage as per manufacturer's instructions and evaluation of alternative methods for estimating tinnitus impact and tinnitus loudness.

Ethics and dissemination This study protocol is approved by the Tallaght Hospital/St. James's Hospital Joint Research Ethics Committee in Dublin, Ireland, and by the Ethics Committee of the University Clinic Regensburg, Germany. Findings will be disseminated to relevant

\section{Strengths and limitations of this study}

- The main strength of this study is that it is a large two-site, triple-blinded, randomised trial that will provide exploratory evidence of the relevance of stimulation parameters on the clinical efficacy of different bimodal stimulation parameters and will inform future trial design.

- The study comprehensively characterises patients for subtyping and this will refine candidature for the intervention.

- Among the limitations of this study are the variability in duration between screening and enrolment and the selection of the investigated stimulation parameters.

- The online recruitment process may inadvertently introduce participant selection bias.

research, clinical, health service and patient communities through publications in peer-reviewed and popular science journals and presentations at scientific and clinical conferences.

Trial registration number The trial is registered on ClinicalTrials.gov (NCT02669069) Pre-results.

\section{INTRODUCTION}

Tinnitus is the perception of sound in the absence of a corresponding external acoustic stimulus. The condition is most commonly referred to as 'ringing in the ears' but symptoms can manifest as buzzing, hissing or sizzling. Tinnitus often coincides with hearing loss and it is commonly believed that hearing loss may be a contributory factor. ${ }^{1}$ While the exact mechanisms responsible for tinnitus are yet to be fully elucidated, it is believed that the reduction in peripheral auditory input, due to hearing loss, results in pathological behaviours that are misinterpreted as sound within the central auditory systems. ${ }^{2}$

Tinnitus has traditionally been treated by means of acoustic stimulation with limited 
success. ${ }^{3}$ Systematic reviews highlight a lack of doubleblind, randomised, controlled studies or quality clinical evidence supporting the efficacy of acoustic stimulation in treating tinnitus. ${ }^{4}$ This has led researchers to investigate approaches to treating tinnitus that go beyond acoustic stimulation.

One approach that has been increasingly investigated in the last decade is invasive and non-invasive neuromodulation of brain structures and networks involved in tinnitus generation. ${ }^{56}$ Neuromodulation is defined as the process of inhibition, stimulation, modification, regulation or alteration of electrical activity in the central, peripheral or autonomic nervous systems. ${ }^{7}$ It is the science of how electrical stimulation can modulate nervous system functionality for therapeutic benefit. Neuromodulation approaches of the central nervous system for the treatment of tinnitus include repetitive transcranial magnetic stimulation, transcranial direct current stimulation and epidural stimulation of temporal, temporoparietal and frontal brain areas. All of these approaches have resulted in reduction of tinnitus handicap in a subgroup of patients. ${ }^{8-11}$ In addition, neuromodulation employing cranial nerve stimulation has been investigated for tinnitus treatment in humans ${ }^{1213}$.

To date, a limited number of uncontrolled pilot studies have been conducted to assess the safety and initial efficacy of neuromodulation employing cranial nerve stimulation for tinnitus. These have included invasive vagus nerve stimulation (VNS), ${ }^{14}$ non-invasive stimulation of the vagus nerve $\mathrm{e}^{1516}$ and non-invasive cervico-trigeminal nerve stimulation (CTNS) ${ }^{12}{ }^{13}$ While VNS demonstrated promising results in animals, ${ }^{17}$ human studies have demonstrated mixed results. ${ }^{14} 1618$ Human studies using non-invasive CTNS have demonstrated promising initial efficacy. ${ }^{12}{ }^{13}$ However, these results should be considered preliminary as the data stems from small pilot studies. The intervention evaluated by Hamilton and colleagues ${ }^{12}$ used synchronised auditory and somatosensory stimulation. However, recent animal research suggests that interstimulus timing intervals may play an important part in the effectiveness of bimodal auditory and somatosensory stimulation on tinnitus. ${ }^{19} 20$ This theory is supported by the findings of a recent 20-patient human pilot study. ${ }^{13}$ Similarly, there is debate in the literature about the choice of auditory stimuli and whether this should stimulate frequency channels associated with sensorineural hearing loss (CTNS approach $)^{12}$ or frequency channels not associated with hearing loss (VNS approach). ${ }^{14}$ Progression to randomised controlled trials (RCTs) with adequately powered sample size is needed. This study protocol represents the first important step towards that goal.

\section{Hypothesis and aims}

The main objectives of the study described here are to investigate the relevance of interstimulus timing and the choices of acoustic and tongue stimuli in order to optimise bimodal neuromodulation parameters for this treatment. Exploratory analyses will be conducted to investigate whether subtypes of patients are differentially responsive to this novel intervention. Safety data will also be collected and reported. Additional feasibility outcomes concern methodological and procedural uncertainties when this novel medical device is prescribed and fitted in a large sample of patients.

\section{METHODS AND ANALYSIS}

\section{Trial design}

The Treatment Evaluation of Neuromodulation for Tinnitus (TENT-A) study is a two-site, randomised, triple-blind, exploratory study examining three different bimodal stimulation parameter sets. The treatment duration is 12 weeks and patients are followed up at 6 weeks, 6 months and 12 months post-treatment cessation. TENT-A will be conducted at two sites: Wellcome Trust-HRB Clinical Research Facility, St. James's Hospital, Dublin, Ireland and Tinnituszentrum Regensburg, University of Regensburg, Germany. The protocol was independently reviewed and approved by Research Ethics Committees of the Tallaght Hospital/St James' Hospital (Ref: 201603-List 11) and the University Clinic Regensburg (Ref: 16-101-0186). The trial sponsor is Neuromod Devices Limited. The trial was registered on ClinicalTrials.gov on 27 January 2016 (Identifier: NCT02669069, Pre-results). The first patient was consented in 22 March 2016 with the last visit planned for May 2018. Our reporting follows standard protocol items for clinical trials defined in the SPIRIT 2013 Statement. ${ }^{21}$

\section{Eligibility criteria}

Eligible patients will be aged 18-70 years at screening; self-report having experienced tinnitus for $>3$ months and $<5$ years; score between 28 and 76 points on the Tinnitus Handicap Inventory (THI), have a Minimum Masking Level (MML) measurement between 20 and 80 decibels hearing level ( $\mathrm{dB} \mathrm{HL}$ ), be able to read and understand English or German (depending on the recruiting centre) and be willing to commit to the duration of the programme.

Potential patients will be excluded if they have pulsatile tinnitus (rhythmical sounds that often beat in time with the heartbeat), tinnitus caused by head or neck injury, or tinnitus resulting from any other neurological condition. Signs of a conductive hearing loss demonstrated by abnormal otoscopy or tympanometry are exclusion criteria; as is a sensorineural hearing loss in either ear of $>40 \mathrm{~dB}$ HL in at least one measurement frequency in the range $0.25-1.00 \mathrm{kHz}$, or of $>80 \mathrm{~dB} \mathrm{HL}$ in at least one measurement frequency in the range $2.0-8.0 \mathrm{kHz}$. Exclusions also include those patients who began wearing a hearing aid within the last 90 days, those with any type of electro-active implantable device (eg, vagal nerve stimulator, cochlear implant or a cardiopacemaker) and those with the following conditions that can be comorbid with tinnitus: Ménière's disease, loudness discomfort level for sounds presented $<30 \mathrm{~dB}$ sensation 
level (SL), temporomandibular joint disorder (TMJ) and anxiety determined by a score $>120$ out of 160 on the state-trait anxiety inventory (STAI) ${ }^{22}{ }^{23}$ Moderate to severe dementia as indicated by a score $<20$ on the mini-mental state examination (MMSE) ${ }^{24}$ will also be a sufficient reason for exclusion. A final set of exclusion criteria based on medical history taken at the screening assessment are: oral piercings, pregnancy, involvement in medicolegal cases, history of auditory hallucinations, any current neurological conditions that may lead to loss of consciousness (eg, epilepsy), current prescription of any drug for a central nervous system pathology and previous use of bimodal neuromodulation devices. Finally, the patient may be excluded if the principal investigator does not deem the candidate to be suitable for the study for other reasons not listed above.

\section{Intervention}

All enrolled patients will receive a proprietary bimodal auditory and somatosensory neuromodulation device (MBT, Neuromod Devices Ltd, Dublin, Ireland), which is a CE-marked Class IIa medical device. Auditory stimulation is delivered through high-fidelity circumaural headphones and comprises a mixture of a wideband noise and sequences of pure tones. Stimulation of the somatosensory system is delivered electrically using an array of 32 transmucosal electrodes on the tongue. Somatosensory stimulation is delivered in the form of biphasic anodic-leading pulses of between 5 and 130 us duration and fixed amplitude. The somatosensory stimulator is arranged so that there is a mapping between the electrodes in the array and frequencies in the tone sequence. The stimuli for each parameter set across the three arms are outlined in table 1.

The auditory stimulus intensity is configured uniquely based on each patient's pure-tone audiometric thresholds in the range $0.25-8 \mathrm{kHz}$, and the patient is afforded limited control over the auditory stimulus intensity of -12 to $+12 \mathrm{~dB}$ in $2 \mathrm{~dB}$ steps during treatment. For patients with $>70 \mathrm{~dB}$ HL hearing loss at any frequency, the upper bound of stimulus intensity control is limited for reasons of safety noise dosage. The treatment device reverts to the default stimulus intensities at the start of each new treatment session. Any adjustments made by the patients to the stimulus intensities are logged in the device's memory for subsequent analysis.

The somatosensory stimulus intensity is configured for each patient at enrolment, based on a calibration procedure that ascertains the patient's threshold of perception. The patient is also afforded limited control over the somatosensory stimulus intensity during treatment.

The device logs the time and date on which the device is in use by the patient, the duration that the electrode array is in contact with the tongue and the intensities of both stimuli.

Individually configured devices will be delivered to the investigator sites with a patient's unique identifier code (UIC) numbers marked on each device and its accessories. Investigators will be extensively trained on fitting the device and instructing patients on its use, per the manufacturer's instructions. Patients will be provided with a quick start guide, an instructions for use (IFU) manual and a link to an instructional video. Before leaving the clinical sites, patients will complete a 30 minute supervised treatment session to ensure that they are comfortable using the device.

\section{Outcomes}

Subjective clinical outcome measures to assess tinnitus impact are the Tinnitus Handicap Inventory $(\mathrm{THI})^{25}$ and the Tinnitus Functional Index (TFI) ${ }^{26}$ The THI provides a measure of the psychological impact of tinnitus, in which 25 items are scored $4 / 2 / 0$ on a categorical scale corresponding to yes/sometimes/no. The global score of the THI has a value between 0 and 100 with the higher scores indicating greater negative functional impact of tinnitus. The TFI assesses a range of functional complaints experienced over the past week. ${ }^{27}$ Each of the 25 items is assessed on an 11-point Likert scale, in which the sum of the scores is normalised to give a global score between 0 and 100, with higher scores indicating greater negative functional impact of tinnitus.

Tinnitus loudness will be assessed by MML, tinnitus loudness matching (TLM) and visual analogue scale (VAS). MML is a psychoacoustic estimate of the lowest level of broadband noise required to minimally mask the patient's tinnitus. ${ }^{28}$ The stimulus is normally presented ipsilaterally (tinnitus ear), or if tinnitus is present in both ears, the stimulus is presented binaurally or to the ear with the

Table 1 Stimulation parameter set for the three parallel arms

Auditory stimulation

Arm 1 Sequence of tones mixed with a broadband noise that is spectrally modified to compensate for any hearing loss

Arm 2 Sequence of tones mixed with a broadband noise that is spectrally modified to compensate for any hearing loss

Arm 3 Sequence of tones mixed with a broadband noise with the Somatosensory pulses are temporally aligned with the

spectral range outside the regions normally associated with tones with varying long delays sensorineural hearing loss

\section{Temporal relationship with somatosensory} stimulation

Somatosensory pulses are synchronised with the tones

Somatosensory pulses are temporally aligned with the tones with varying short delays 
predominate sound. TLM will be assessed by presenting a $1 \mathrm{kHz}$ tone $\mathrm{e}^{29}$ contralateral to the predominant tinnitus ear or if tinnitus is equally loud in both sides or localised in the head, the stimuli will be presented to the ear with better hearing or randomly selected. The stimulus will be increased in $1 \mathrm{~dB}$ increments until the patient confirms that it is equal in loudness to their tinnitus. Finally, a VAS will be employed for patients to rate the current loudness of their tinnitus, with 0 equating to 'not loud at all' and 10 equating to 'extremely loud', as the endpoints anchors. ${ }^{30}$

Safety data on adverse events (AEs) and serious adverse events (SAEs) will be captured throughout the trial. An $\mathrm{AE}$ is defined as any unfavourable and unintended sign, symptom or disease, temporarily that may or may not be related to the medical device. It will be rated as minor, major or serious and related or unrelated to the device by the TENT-A Medical Review Board. An SAE is defined as an $\mathrm{AE}$ that led or might have led to the death or serious deterioration in the state of health of a patient. Treatment-related AEs are those judged by the principal investigator at each site to be possibly caused by the treatment under investigation. The principal investigator will remain vigilant for signs of possible treatment-related changes in oral health (eg, irritations in the oral cavity or discomfort between the tongue tip and dental retainers or metal fillings) and the impact of tinnitus (indicated by the THI and TFI).

Stopping criteria are defined as patients demonstrating a worsening in THI and MML of an increase in THI of 7 points and an increase in MML of $5.3 \mathrm{~dB}$. Treatment-related changes in hearing thresholds that are considered an $\mathrm{AE}$ are a deterioration from screening to endpoint of $15 \mathrm{~dB}$ in a minimum of two adjacent test frequencies $(0.25-8 \mathrm{kHz})$ in either ear that cannot be explained by conductive hearing problem or a recent excessive noise exposure.

Additionally, feasibility outcomes include: reasons for exclusion at the screening visit, number of patients who were eligible at the screening appointment but declined to participate further, number of patient withdrawals after device fitting, proportion of incomplete patient datasets at each scheduled visit, patients' compliance with the device usage as per manufacturer's instructions and comparisons of alternative methods for measuring the impact of tinnitus and for estimating tinnitus loudness.

Compliance data will be extracted from log files saved on the device. For feasibility analysis, compliance rate will be expressed as a percentage of logged usage relative to (i) the expected compliance as per the intended use for the device (a total of 84 hours over the 12-week period), and to (ii) a predefined minimum acceptable compliance threshold (defined as at least 3 hours average usage within a 1-week period, corresponding to a sum total of 36 hours of treatment).

\section{Recruitment}

Patients will be primarily recruited via media advertising and dedicated trial websites in both clinical locations.
Advertisements on regional and national radio stations and in regional and national newspapers will invite individuals with tinnitus, who are interested in participating in a clinical study for tinnitus, to register their interest on dedicated recruitment websites. The recruitment website provides information on the study and how to proceed with registration. Once they register their interest, candidates will be provided with a UIC and an accompanying personal identification number and be directed to an eligibility assessment website. The eligibility assessment comprises an online survey, hosted by SurveyGizmo, where those interested can find out about the requirements of participating in the study. Candidates will answer a scripted set of general prescreening questions on age, duration of tinnitus, oral piercings, other current medical conditions including TMJ disorder and Ménière's disease and involvement in medicolegal cases. This is not part of the formal screening because no personal or medical details will be taken, but it is intended to manage the large numbers of candidates expected to respond to the advertising campaign and anticipated high screen failure rate. Candidates who meet the inclusion criteria at this stage will be provided with a patient information leaflet and informed consent via email or post and invited to a screening visit at the local site.

\section{Patient timeline}

The schedule of clinical research activities is illustrated in table 2 and briefly comprises seven visits to the clinical site, plus two telephone calls during the period of device usage. The various assessments are completed by a multidisciplinary team including: audiologists, medical doctors, physiotherapist, research nurses and research associates.

The initial objective of the screening visit is to obtain written informed consent to determine whether the patient satisfies the remaining eligibility criteria and to obtain initial outcome measures, patient characteristics and audiological profile. This information is employed in the subtype classification of patients, the stratified random allocation process and for device configuration, described below.

At the enrolment and device fitting visit, a physiotherapist (Dublin) or medical doctor (Regensburg) conducts a comprehensive assessment comprising a set of 25 predefined cranial manipulations designed to diagnose somatic tinnitus. ${ }^{31}$ We define somatic tinnitus where a patient reports that at least one of the somatic manipulations reliably produces a change in the psychoacoustic characteristics of their tinnitus (e.g., in pitch, loudness, localisation, temporal properties and so on). Assessments of outcome measures are repeated at the enrolment visit. Other elements of the visit include an oral health examination and device training and deployment. After completing an on-site supervised treatment session, patients return home and self-administer the treatment. Patients will be instructed to use the device for two daily $30 \mathrm{~min}$ sessions over a 12-week period. 


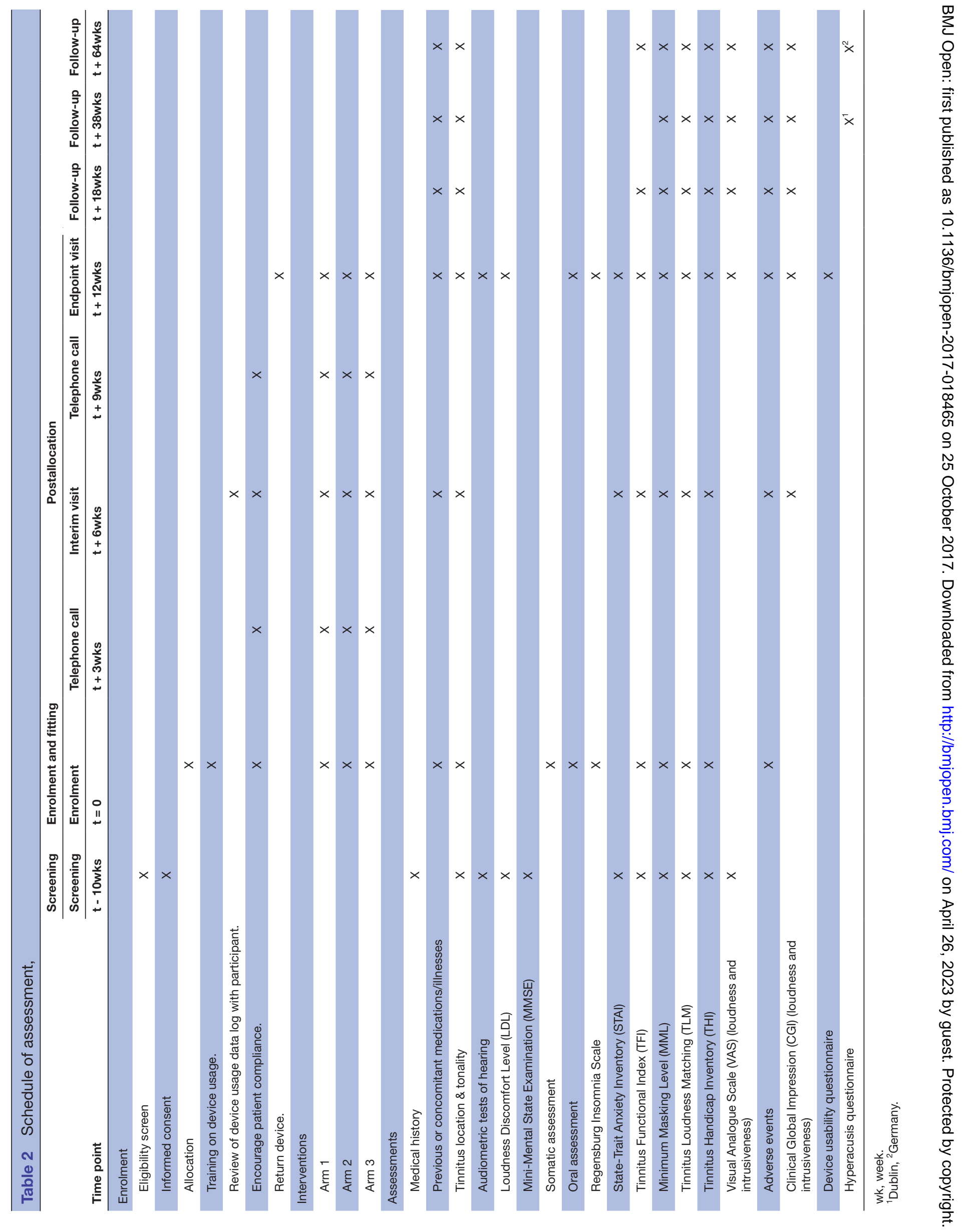




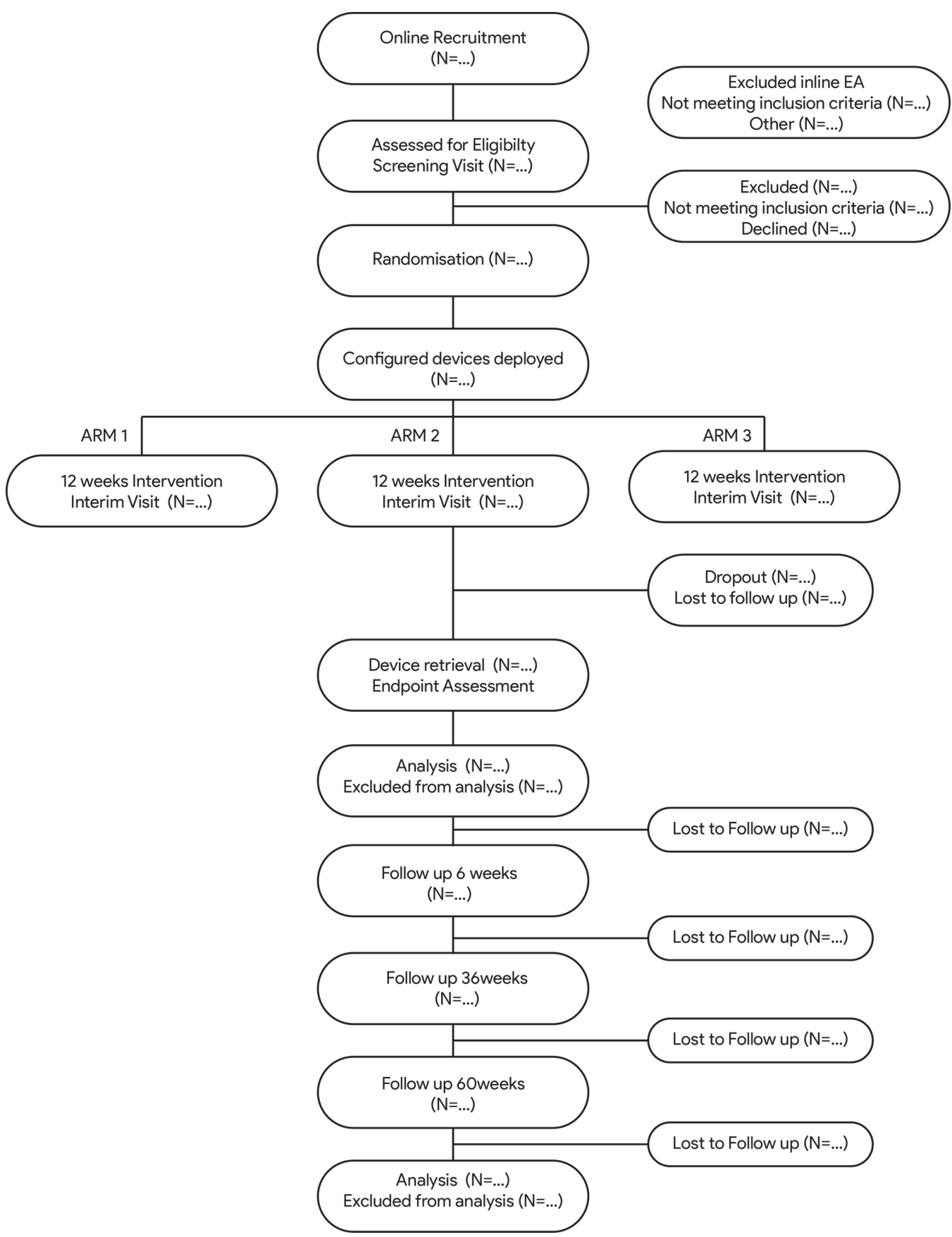

Figure 1 Anticipated flow of participants through the TENT-A study. TENT-A, Treatment Evaluation of Neuromodulation for Tinnitus.

These sessions can be continuous or at different times of the day.

Assessment of outcome measures and safety information are collected at the interim visit, half way through the 12-week treatment. Compliance will also be assessed and reviewed at the interim visit. Investigators will review the device usage log. Patients with acceptable compliance will be encouraged to continue and patients with poor compliance will be encouraged to improve. Compliance phone calls will be conducted at weeks 3 and 9 to encourage patients to continue with device usage and address any technical issues that patients may be having.
The purpose of the endpoint visit is to repeat outcome measures assessments, the oral health examination, an exit interview and retrieve the device. Three follow-up visits will be conducted to assess the post-cessation effects of this intervention.

\section{Sample size}

The study is powered for a between-arm clinically significant difference in the mean THI scores from baseline to endpoint, where the reported clinically significant change in THI is 7 points. ${ }^{32}$ The assumed sample SD is 12.7 points, as elucidated from a previous study using 
similar technology, ${ }^{12}$ resulting in an effect size of 0.55 . The sample size calculations were performed using MATLAB 2016a, assuming a two-sided significance level of 0.016 ( 0.05 split equally between the three interarm pairwise t-tests), and power of $90 \%$, resulting in a total of 91 patients to be enrolled in each treatment arm, or 114 patients per arm to account for an expected drop-out rate of $20 \%$. In total, 342 patients will be required across the three arms of the study, split 80:20 between the Dublin and Regensburg sites, respectively.

\section{Allocation}

Eligible patients will be randomised, in equal proportions, between the three parallel arms (see figure 1). Stratified randomisation will be performed to balance the influence of several baseline covariates in the post-hoc analyses. The stratification covariates are chosen based on the investigator's research objectives, namely to elucidate relative treatment effects on patients with tinnitus with varying underlying characteristics. Allocation of patients will be stratified across the three intervention arms according to four binary categories applied in a stepwise manner: (i) tinnitus tonality, (ii) sound level tolerance (using loudness discomfort level of $<60 \mathrm{~dB}$ SL as an indicator for hyperacusis), (iii) 'normoacoustic' (defined as pure-tone thresholds of $\leq 20 \mathrm{~dB}$ HL in all audiometric test frequencies between 0.25 and $8 \mathrm{kHz}$ in both ears) and (iv) presence of a noise-induced audiometric profile (defined as a dip in pure-tone hearing thresholds of $10 \mathrm{~dB}$ or more in any frequency in the 3 to $6 \mathrm{kHz}$ range in either ear). These covariates are not mutually exclusive, so priority during stratified randomisation will be given to the least prevalent covariates based on candidate characteristics from the screening phase of the study.

The stratification and randomisation will be performed adaptively (Minimisation), ${ }^{33}$ whereby the probability of assignment to a treatment intervention changes as the imbalance within the relevant stratum increases. Dice rolls emulated in MATLAB's Mersenne Twister algorithm (V.2016a) will be used with the randomisation seed set to the date each new block of patients is randomised.

\section{Data collection methods}

All data will be collected electronically using a validated electronic case report form (eCRF) application. Patient data collected at all stages of the trial will be entered into the eCRF using UIC's assigned to patients at recruitment phase. All investigators and patients will be blinded to allocation arm and no allocation information will be contained in the eCRF. The data monitors will be able to remotely view the data in the eCRF to monitor safety data.

\section{Statistical methods}

The main indicators of clinical efficacy for the three different parameter sets are two patient-reported outcomes (THI and TFI) after 12 weeks of intervention. Baseline outcome measures are computed as the average scores at the screening and enrolment visits. Clinical efficacy will be explored for a series of patient subtypes, split by the stratification variables, while evidence for sustained effects on the psychological and functional impact of tinnitus will be followed up for 12 months. Additional feasibility measures to inform future trial design include: reasons for exclusion, completeness of data collection, attrition rates and patients' adherence to the device usage as per manufacturer's instructions. These will be collated using descriptive statistics and reported as percentages. We will also evaluate the alternative methods for estimating tinnitus impact and tinnitus loudness, as far as possible using psychometric criteria defined by the COnsensus-based Standards for the selection of health Measurement INstruments completed in line (COSMIN) checklist. ${ }^{34}$

Efficacy analyses will focus on investigating (i) between-arm changes in the THI and TFI outcome measures from baseline to endpoint, and (ii) within-arm changes in THI and TFI outcome measures from baseline to endpoint, for the full cohort and then sequentially testing the subtypes described above using serial gatekeeping to control the family-wise error rate at the 0.05 significance level. The between-arm analyses will be based on an intention-to-treat estimand and tested with multiple regression using baseline scores as a covariate. Missing data will be handled by using Markov chain Monte Carlo multiple imputation methods ${ }^{35}{ }^{36}$. The within-arm analyses will be based on a per-protocol estimand and tested with paired two-tailed t-tests. The use of per-protocol estimand will ensure that the changes in outcome measures within a particular treatment arm are reflective of real-use scenarios, that is, where the patients use the treatment as directed. The threshold for inclusion in the per-protocol analysis is set at the predefined minimum acceptable compliance threshold previously described. Additional exploratory efficacy analyses shall be conducted in order to ascertain treatment effects from baseline to interim (i.e. 6 weeks of treatment), and to evaluate any sustained efficacy by analysing changes in efficacy outcome measures from endpoint to the three follow-up assessments (i.e. at 18, 36 and 60 weeks after device fitting).

Safety analyses will be performed by evaluating the incidence of adverse events, classified as treatment or non-treatment related, and further sub-classified as minor, major and serious. Adverse events will be recorded proactively by monitoring significant changes in THI, TFI, MML; hearing thresholds and oral health; and reactively by documenting any adverse events reported by patients during the study. All adverse events will be analysed for trends, and statistical tests for significant between-arm differences will be conducted.

Efficacy and safety data analysis will be conducted in compliance with the Consolidated Standards of Reporting Trials guidelines for RCTs ${ }^{37}$ using the SAS software package. 


\section{Dissemination}

Findings will be disseminated to relevant research, clinical, health service and patient communities through publications in peer-reviewed and popular science journals and presentations at scientific and clinical conferences.

\section{DISCUSSION}

This paper outlines the protocol for a multisite, randomised, triple-blind, exploratory study designed to explore the effects of different bimodal stimulation parameter sets across a number of tinnitus subtypes in a range of tinnitus clinical subdomains. The results of this study will inform the design of future triple-blind randomised control trials. The main objective is to determine an optimised bimodal stimulation parameter set, but we will also explore which patient characteristics might best predict therapeutic benefit to this treatment. We anticipate that this could lead to improved targeted intervention options for people with chronic subjective tinnitus.

This study is timely for several reasons. First, completing this exploratory trial will be important in determining any feasibility challenges and will be used to estimate the time, resources and sample size required for future confirmatory trials. Second, findings from this study could potentially inform the acceptability of bimodal stimulation in the wider population. There is a recognised need for evidenced therapeutic options that reduce or alleviate the symptoms of tinnitus instead of simply helping sufferers to manage the cognitive, emotional and behaviour impacts of their long-term condition. ${ }^{3}$ Third, low-quality clinical trial design and reporting has been identified as a major barrier to developing effective tinnitus therapies and standards of practice have been proposed. ${ }^{38-40}$ The study design and protocol description are in line with those recommendations.

\author{
Author affiliations \\ ${ }^{1}$ Neuromod Devices Limited, Dublin, Ireland \\ ${ }^{2}$ National Institute for Health Research Nottingham Biomedical Research Centre, \\ Nottingham, UK \\ ${ }^{3}$ Otology and Hearing Group, Division of Clinical Neuroscience, University of \\ Nottingham, Nottingham, UK \\ ${ }^{4}$ Lab for Clinical and Integrative Neuroscience, School of Behavioral and Brain \\ Sciences, The University of Texas at Dallas, Richardson, Texas, USA \\ ${ }^{5}$ Department of Psychiatry and Psychotherapy, University of Regensburg, \\ Regensburg, Germany \\ ${ }^{6}$ Interdisciplinary Tinnitus Center of the University of Regensburg, Regensburg, \\ Germany \\ ${ }^{7}$ ENT, Tallaght Hospital, Dublin, Ireland \\ ${ }^{10}$ ENT, St. James's Hospital, Dublin, Ireland \\ ${ }^{8}$ Department of Medicine, Trinity College, Dublin, Ireland
}

Contributors SDA, CH, SH and BC: conceived of the study. SDA, $\mathrm{CH}$ and SH: initiated the study design. SH: provided statistical expertise in clinical trial design. BL, SV and DAH: are members of the Science Advisory Board for Neuromod Devices Ltd and contributed to the Clinical Investigation Plan and the Statistical Analysis Plan, on which the protocol is based. BL and BC: principal investigators at the two trial sites. All authors: contributed to the refinement of the study protocol and approved the final manuscript. SDA: submitted the manuscript for publication.

Funding This work was supported by Neuromod Devices Ltd.
Competing interests $\mathrm{SD}, \mathrm{CH}, \mathrm{SH}$, and $\mathrm{BC}$ are employees and shareholders of the Sponsor. BL, SV and DAH act as paid consultants for the Sponsor.

Ethics approval Tallaght Hospital / St. James's Hospital Joint Research Ethics Committee in Dublin, and Ethics Committee of the University Clinic Regensburg, Germany.

Provenance and peer review Not commissioned; externally peer reviewed.

Open Access This is an Open Access article distributed in accordance with the Creative Commons Attribution Non Commercial (CC BY-NC 4.0) license, which permits others to distribute, remix, adapt, build upon this work non-commercially, and license their derivative works on different terms, provided the original work is properly cited and the use is non-commercial. See: http://creativecommons.org/ licenses/by-nc/4.0/

(c) Article author(s) (or their employer(s) unless otherwise stated in the text of the article) 2017. All rights reserved. No commercial use is permitted unless otherwise expressly granted.

\section{REFERENCES}

1. Lockwood AH, Salvi RJ, Burkard RF. Tinnitus. N Engl J Med 2002;347:904-10.

2. Eggermont JJ, Roberts LE. The neuroscience of tinnitus. Trends Neurosci 2004;27:676-82.

3. Hoare DJ, Kowalkowski VL, Kang S, et al. Systematic review and meta-analyses of randomized controlled trials examining tinnitus management. Laryngoscope 2011;121:1555-64.

4. Hoare DJ, Edmondson-Jones M, Sereda M, et al. Amplification with hearing aids for patients with tinnitus and co-existing hearing loss. Cochrane Database Syst Rev 2014;1:CD010151.

5. Langguth $B$, De Ridder D. Tinnitus: therapeutic use of superficial brain stimulation. Handb Clin Neurol 2013;116:441-67.

6. Vanneste S, De Ridder D. Noninvasive and invasive neuromodulation for the treatment of tinnitus: an overview. Neuromodulation 2012;15:350-60.

7. Krames ES, Peckham PH, Rezai AR, et al. What is neuromodulation? In: Krames ES, Peckham PH, Rezai AR, eds. Neuromodulation. San Diego: Academic Press, 2009:3-8.

8. De Ridder D, Song JJ, Vanneste S. Frontal cortex TMS for tinnitus. Brain Stimul 2013;6:355-62.

9. Folmer RL, Theodoroff SM, Casiana L, et al. Repetitive transcranial magnetic stimulation treatment for chronic tinnitus: a randomized clinical trial. JAMA Otolaryngol Head Neck Surg 2015;141:716.

10. Rabau S, Van Rompaey V, Van de Heyning P. The effect of transcranial direct current stimulation in addition to tinnitus retraining therapy for treatment of chronic tinnitus patients: a study protocol for a double-blind controlled randomised trial. Trials 2015; $16: 514$

11. De Ridder D, Vanneste S, Kovacs S, et al. Transcranial magnetic stimulation and extradural electrodes implanted on secondary auditory cortex for tinnitus suppression. J Neurosurg 2011;114:903-11.

12. Hamilton C, D'Arcy S, Pearlmutter BA, et al. An investigation of feasibility and safety of bi-modal stimulation for the treatment of tinnitus: an open-label pilot study. Neuromodulation 2016;19:832-7.

13. Shore S, Marks K, Martel DT, et al. Auditory-Somatosensory Stimulation Alleviates Tinnitus in Human Subjects. Baltimore: Proc. ARO 40th Annual Mid-winter meeting, 2017.

14. De Ridder D, Vanneste S, Engineer ND, et al. Safety and efficacy of vagus nerve stimulation paired with tones for the treatment of tinnitus: a case series. Neuromodulation 2014;17:170-9.

15. Kreuzer PM, Landgrebe M, Resch M, et al. Feasibility, safety and efficacy of transcutaneous vagus nerve stimulation in chronic tinnitus: an open pilot study. Brain Stimul 2014;7:740-7.

16. Lehtimäki J, Hyvärinen $P$, Ylikoski $M$, et al. Transcutaneous vagus nerve stimulation in tinnitus: a pilot study. Acta Otolaryngol 2013;133:378-82.

17. Engineer ND, Riley JR, Seale JD, et al. Reversing pathological neural activity using targeted plasticity. Nature 2011;470:101-4.

18. Kreuzer PM, Landgrebe M, Husser $\mathrm{O}$, et al. Transcutaneous vagus nerve stimulation: retrospective assessment of cardiac safety in a pilot study. Front Psychiatry 2012;3:70.

19. Koehler SD, Shore SE. Stimulus timing-dependent plasticity in dorsal cochlear nucleus is altered in tinnitus. J Neurosci 2013;33:19647-56.

20. Markovitz CD, Smith BT, Gloeckner CD, et al. Investigating a new neuromodulation treatment for brain disorders using synchronized activation of multimodal pathways. Sci Rep 2015;5:9462. 
21. Chan AW, Tetzlaff JM, Gøtzsche PC, et al. SPIRIT 2013 explanation and elaboration: guidance for protocols of clinical trials. BMJ 2013;346:e7586.

22. Spielberger CD, Gorsuch RL, Lushene R, et al. Manual for the statetrait anxiety inventory. Palo Alto, CA: Consulting Psychologists Press, 1983.

23. Kvaal K, Ulstein I, Nordhus IH, et al. The spielberger state-trait anxiety inventory (STAI): the state scale in detecting mental disorders in geriatric patients. Int J Geriatr Psychiatry 2005;20:629-34.

24. Folstein MF, Folstein SE, McHugh PR. "Mini-mental state". A practical method for grading the cognitive state of patients for the clinician. J Psychiatr Res 1975;12:189-98.

25. Newman CW, Jacobson GP, Spitzer JB. Development of the tinnitus handicap inventory. Arch Otolaryngol Head Neck Surg 1996;122:143-8.

26. Meikle MB, Henry JA, Griest SE, et al. The tinnitus functional index: development of a new clinical measure for chronic, intrusive tinnitus. Ear and Hearing 2012;33:153-76.

27. Fackrell K, Hall DA, Barry JG, et al. Psychometric properties of the Tinnitus Functional Index (TFI): Assessment in a UK research volunteer population. Hear Res 2016;335:220-35.

28. Henry JA, Meikle MB. Psychoacoustic measures of tinnitus. J Am Acad Audiol 2000;11:138-55.

29. Henry JA, James KE, Owens K, et al. Auditory test result characteristics of subjects with and without tinnitus. J Rehabil Res Dev 2009;46:619.

30. Adamchic I, Langguth B, Hauptmann C, et al. Psychometric Evaluation of Visual Analog Scale for the Assessment of Chronic Tinnitus. Am J Audiol 2012;21:215.
31. Somatic LRA. craniocervical) tinnitus and the dorsal cochlear nucleus hypothesis. Am. J. Otolaryngol 1999;20:351-62.

32. Zeman F, Koller M, Figueiredo R, et al. Tinnitus handicap inventory for evaluating treatment effects: which changes are clinically relevant? Otolaryngol Head Neck Surg 2011;145:282-7.

33. Pocock SJ, Simon R. Sequential treatment assignment with balancing for prognostic factors in the controlled clinical trial. Biometrics 1975;31:103-15.

34. Mokkink LB, Terwee CB, Patrick DL, et al. The COSMIN checklist for assessing the methodological quality of studies on measurement properties of health status measurement instruments: an international Delphi study. Qual Life Res 2010;19:539-49.

35. Rubin DB. Multiple imputation for nonresponse in surveys. John Wiley \& Sons, 2004

36. Schafer JL. Analysis of incomplete multivariate data. CRC press, 1997.

37. Schulz KF, Altman DG, Moher D. CONSORT 2010 statement: updated guidelines for reporting parallel group randomised trials. PLoS Med 2010;7:e1000251.

38. Hall DA, Haider $\mathrm{H}$, Kikidis $\mathrm{D}$, et al. Toward a global consensus on outcome measures for clinical trials in tinnitus: report from the first international meeting of the COMiT Initiative, November 14 2014, Amsterdam, The Netherlands. Trends Hear;2015: 2331216515580271.

39. Hall DA, Haider H, Szczepek AJ, et al. Systematic review of outcome domains and instruments used in clinical trials of tinnitus treatments in adults. Trials 2016;17:270.

40. Landgrebe M, Azevedo A, Baguley D, et al. Methodological aspects of clinical trials in tinnitus: A proposal for an international standard. J Psychosom Res 2012;73:112-21. 